\title{
Characterization and molecular diagnosis of HIV/ADIS and its application
}

\author{
Mazen Eidah Alqurashi and Ghassan Mohammed Abusabaa \\ Molecular Biology laboratory at King Fahd General Hospital, Jeddah City, Saudi Arabia \\ Correspondence Author: Mazen Eidah Alqurashi, Molecular Biology laboratory at King Fahd General Hospital, Jeddah City, Saudi Arabia. \\ Email: mealqurashi@moh.gov.sa
}

Received date: 12 May 2019, Accepted date: 24 September 2019, Online date: 5 October 2019

Copyright: (c) 2019 Mazen Eidah Alqurashi and Ghassan Mohammed Abusabaa. This is an open-access article distributed under the terms of the Creative Commons Attribution License, which permits unrestricted use, distribution, and reproduction in any medium, provided the original author and source are credited.

\begin{abstract}
HIV laboratory diagnosis of children under 18 months of age requires two positive PCR results for HIV, each of which is tested separately according to national guidelines for HIV testing. in children on June 1, 2015 (help positive PCR result for HIV due to detectable HIV virus in HIV diagnosis, if only one sample is present). The HIV PCR result can be positive, negative or uncertain. The uncertainty of the PCR-PCR results indicates that the test is not significant it is not clearly positive or negative and requires immediate Additional testing, to determine if the child is infected with HIV or not, and RETURNS. Repeat HIV-PCR and HIV viral load: The tests should be performed for each emergency and the patient should be treated accordingly. A for treatment of uncertain cases of the initial HIV-PCR and scenario B for the treatment of PCR cases. for confirmation of unsecured PCR). Infants with an unclear or conflicting HIV diagnosis. The aim of this work an overview of HIV/ADIS characterization and molecular diagnosis of and their application.
\end{abstract}

Keywords: HIV, ADIS, characterization, molecular, PCR, diagnosis, application

\section{INTRODUCTION}

Laboratory diagnosis of HIV in adults: a review of modern methods. Approximately 34 million people are infected with human activities and viral activity (HIV) worldwide, including 1.2 million in the United States. However, it is estimated that $20 \%$ of HIV-infected people in the US do not yet know the diagnosis [1]. HIV testing guidelines in the United States. They were originally intended for people with a history of sexual behavior in the context of high-risk sexually transmitted infections, blood transfusions between 1978 and 1985 or intravenous drug use [2]. However, in recent years, efforts have been made at the national level to develop the HIV test. In 2006, the Center for Disease (CDC) launched the Multiplication of HIV Testing initiative, strengthening HIV testing and reducing the number of infections diagnosed. The CDC's current recommendation is for the routine screening of people aged 13 to 64 for HIV infection [3] In countries with limited resources, the situation was even more difficult. 2010 showed population surveys conducted by the World Health Organization, more than $69 \%$ of people in 10 countries in sub-Saharan Africa were not aware of their HIV status [4], and awareness and HIV testing were also limited in the United States. The CDC estimates that national data on HIV incidence in the United States each year more than 50,000 cases of HIV infections affect [5]. Ideally, the HIV test for expansion not only helps to diagnose HIV, but also the most common HIV testing during acute HIV infection is called a defined period. the clinical syndrome associated with a strong replication of the virus and the phase between the appearance of detectable p24 or R NA detectable by anti-HIV antibodies [6]. Studies have shown that the rate of infectivity is high the acute phase of infection, HIV [7], leads to an increase in transmission during this phase and regardless of the viral load $[8,9]$. Seropositive PCR followed by seronegative PCR and undetectable HIV viral load) should be discussed and discussed in detail by an interdisciplinary team.

The estimates of symptomatic acute HIV vary in Emergency care by a doctor and a pathologist. HIV and clinical surveillance are repeated until HIV status is established. It is important to remember that children are not considered with infected HIV. At least the repeated test takes place at least 4 weeks after the prophylactic baby administered (or already dropped ART) and six weeks after "permission to breastfeed". For the care of the competent lawyer mother / responsible for the uncertain exit responsible person is of utmost importance for a successful follow-up to ensure and make a definitive diagnosis.

The global health community is increasingly calling on the PDF to simplify and improve efficiencies, without compromising the quality of care provided to patients in the diagnosis of HIV/AIDS. At the same time, there is a need to significantly increase the level of access to reliable and high-quality diagnostic environments with limited resources, one to facilitate early diagnosis and treatment of HIV/AIDS. Among the various tests for initial diagnosis, evaluation and ongoing surveillance of HIV, the most serious problems related to access and improved effectiveness are CD4, viral load and early diagnosis of infants. This report reviews advanced diagnostic platforms and pipeline technologies for these three key tests. For all of them, most current testing capabilities are also not flat: forms of laboratory work on complex instruments, specialized laboratory space and requiring a trained laboratory technician. In many cases, laboratory tests are expensive. In almost all cases, there is a need for selective patient transport access systems in suburban and rural areas [9].

HIV test at birth using PCR in terms of service and regular HIV DNA: a pilot feasibility study in Kenya

In 2015, about 110,000 new cases of HIV infection with the human immunodeficiency virus (HIV) were recorded in the 21 target countries of the Global Plan [10]. The survival child and the success of antiretroviral therapy (ART) in HIV-infected children at an early stage are early in life [11]; Therefore, early 
Citation: Mazen Eidah Alqurashi, et al, 2019. Characterization and molecular diagnosis of HIV/ADIS and its application. Journal of Applied Sciences Research., 15(5): 28-33. DOI: $10.22587 /$ jasr.2019.15.5.6

detection of HIV, diagnosis and rapid onset of antiretroviral therapy is essential. In 2015, there were about 6,600 HIV-infected children in Kenya, 55 percent less than in 2009, but in Kenya, the number of HIV-infected children who have been testing themselves for two months. routine PCR (PCR), has stagnated by nearly $50 \%$ since increased $[12,13]$. One of the barriers to successful early diagnosis in children is the waiting time to get the test results. The sequence of DIP services includes many urgent steps required by coordination between the service provider, the diagnostic laboratory, and the parent/manager ("nut"). Kenya's standard algorithm for digital identity includes the introduction of tests for mothers with children. Sampling, transport and laboratory analysis; transmit the results to health workers and caregivers; and the relationship with treatment, including the prevention of opportunistic infections in all HIV-infected children and antiretroviral therapy in children with HIV [14]. All stages are associated with delays and patient loss during follow-up [14 - 16]. Long-distance travel for clinical care is a logistical barrier and additional resource for effective treatment of HIV infection [17]. Innovative strategies to accelerate the provision of adequate care to HIVinfected mothers include starting the disease at birth and then rationalizing the age of 6 weeks and the cascade of diseases [18]. Although these approaches are promising, limited resources should be considered for all service providers (service providers, laboratories and mothers). This is probably a checkpoint at birth or birth with Add Technology Standard of Care (SOC) or at a point of treatment (POC), which requires more load and the ability to work, at the both in the clinic and in the laboratory. team [19] and possibly more trips to the clinic of mothers and babies. Conventional testing in HIV-exposed infants did not start until 6 weeks of age because the sensitivity of the PCR test with DNA-PCR is lower in infants and neonates (68\%). 88\% at birth and 4 weeks [20]); During childbirth (CAP), an HIV infection is not detected immediately after birth and a six-week delay coincides with visits for the vaccine. Infants are infected with HIV. For the first time in about six weeks, they were often treated with antiretrovirals at the median age of 16 and 24 and a half weeks of interlocutory treatment [21-24 December], which also corresponds to the 8 weeks. -12 weeks [25].

In Kenya, $61 \%$ of pregnant women give birth in a health centre and $20 \%$ of newborns at home are the subject of a postnatal survey within one week of birth [26]. Therefore, testing at birth or at first clinical contact may accelerate the onset of antiretroviral therapy in HIV-infected intrauterine infants. Recent recommendations from WHO and Kenya's Ministry of Health [27, 28] reflect the consensus that HIV testing at birth can improve outcomes in infants with HIV, even with lower sensitivity for HIV. neonatal specimens [29, 30]. Kenya's guidelines include, in addition to other six-week PCR HIV tests at 6 months and 12 months, DNA testing for HIV at birth for HIV-infected births. However, a birth test ( 0 to 2 weeks) prior to national deployment requires pilot data. In 2016 , the WHO pre-qualified two diagnostic tests to detect HIV RNA in whole blood (Xpert orchestrates an HIV-1 agony for GeneXpert, manufactured by AB Cepheid, Alereq an HIV-1/2, manufactured by Abbott). Both tests are high-quality, real-time reverse-transcription PCR using table-top instruments suitable for clinical operation and not requiring in-depth technical training [31,32].

The published estimates of the first Xpert HIV-1 or QUAL Alere HIV-1/2 operational tests have already been carried out in the laboratory [31-34], which show good sensitivity (93.3 to $98.7 \%$ ) and specificity (100\%). with children's motif. In the test, the POC system provides the optimal value for accelerating the EID when the clinician is performed in the clinic. Several studies involving Xpert HIV1 Kutch [35, 36] and Alere d HIV - 1/2 [37 - 38] have been conducted in medical educators in medicine. The sensitivity and specificity values were like the reported laboratory qualification studies, but in some cases, the field study indicators allowed for further detection of errors $[35,38]$. Recent studies of many HIV-infected babies show a close relationship between SOC and two EOC testing technologies $[35,36]$. Although the analytical properties of these POC technologies have been characterized as a study area, the scale and utility in Kenya and other resource-constrained countries require implementation studies. stable in different clinical conditions. At present, there are no adequate data for the strategic implementation of the Xpert and Alere q POC control or birth control platform points in Kenya. Here we present a pilot study protocol on the feasibility and the preliminary effects of the introduction of birth as assessment tests for DIP complement services and two parallel DNA-DNA POC platforms - PCR in the laboratory to evaluate in this feasibility study in Kenya, there is a schedule for implementing new tests, birth and two platforms to determine the diagnosis of newborns at the pilot scale. ROS HIV Xpert and Alere q platforms in four public hospitals in Kenya were tested and tested for six weeks. This 18-month study was conducted in addition to the parent study (R01HD076673) for the implementation and evaluation of an HIV testing system for babies (HIT system) started [39]. The four hospitals designated as research sites are public hospitals with average and higher patient volumes and geographical diversity: two in Kisumu County, one in Nakuru County and Mombasa County. The estimated rate of mother-to-child HIV transmission in these three countries was 20\%,5\% and 18\% in 2015 [40]. Phase 1 is a six-month training phase in which we will conduct quality interviews with parents, caregivers and community members to accept or retain obstacles of the attitude of evaluation of the actors to test the births of the tests of performance. Comments are accustomed to exploitation plans in terms of feasibility and acceptability to be clarified. Phase 2 consists of a two-month feasibility study for two new POC test strategies. The 12-month implementation period gives suppliers the time to introduce new methods and monitor variations in uptake or acceptance over this long period. An HIV-POC test is performed with standard HIV-based laboratory DNA-PCR, to prepare samples at birth ( 0 to 2 weeks) and after 6 weeks ( 4 to <24 weeks). Phase 1: Formative Research During Phase 1 , key stakeholders are invited to provide information that can guide operational planning to optimize the implementation, acceptance, and feasibility of services at each site. research. Approximately 25 educational interviews are conducted with each parent, provider and community member at each site. Only 100 interviews. In interviews with parents (HIV-positive mothers who have already experienced the disease) and their male partners (if applicable) and the mother who has already established HIV status ( $\mathrm{n}=10$ per site), the consequences for the baby and her are discussed Family (confidentiality/disclosure, acceptance of the chosen time). Tests and results as well as the care of the partner).

Interviews with suppliers who participated in POC testing at each facility (maternal, child and maternal health care, teachers, technicians and medical antiretroviral therapy, approximately $\mathrm{n}=5$ for each site) focused on training issues. Site-specific logistics, training and patient counseling, as well as the staff and resources needed for implementation. We will also conduct interviews with community members (health workers respected by traditional midwives, community cooks, and religious leaders, approximately $\mathrm{n}=10$ for each site) in a rural community in the area of influence of the community. each hospital to generate relationships and suggestions on the difficult potential for regular HIV testing with EFPs in the communities. The consent form will be obtained in person prior to the interview. Participants receive 500 Kenyan shillings to compensate for travel time. The recordings will be recorded analytically for later transcription, translation, coding and sound analysis. The transcripts are coded independently by two members of the study group to identify emerging priorities and issues. The coding differences are resolved by group consensus. We will develop a codebook with typical examples for each subject, considering the frequency and distribution of topics on a wide range of topics. The research team will review the topics to inform the POC pilot [41].

Intervention. Design Studio We have publicly tested for 12 months in four POC hospitals and fertility tests. Four subjects will use a randomized randomized system on one of the two POC systems (Xpert Agon with HIV-1 for HIV-RNA or Alere q for HIV-half Detect). SOC diagnostic tests (PCR test for HIV DBS DNA samples) will be organized in parallel at all study sites, also at the newly introduced time at birth tested. The establishment, authorization, and consent of pregnant women infected with HIV or mothers of HIV-infected infants treated in teaching hospitals are invited to participate in a pilot project. Women can register via PMTCT during pregnancy. during birth, during delivery; or MCH during your first postnatal visit. Women must be at least 18 years old and be infected with HIV [41]. 


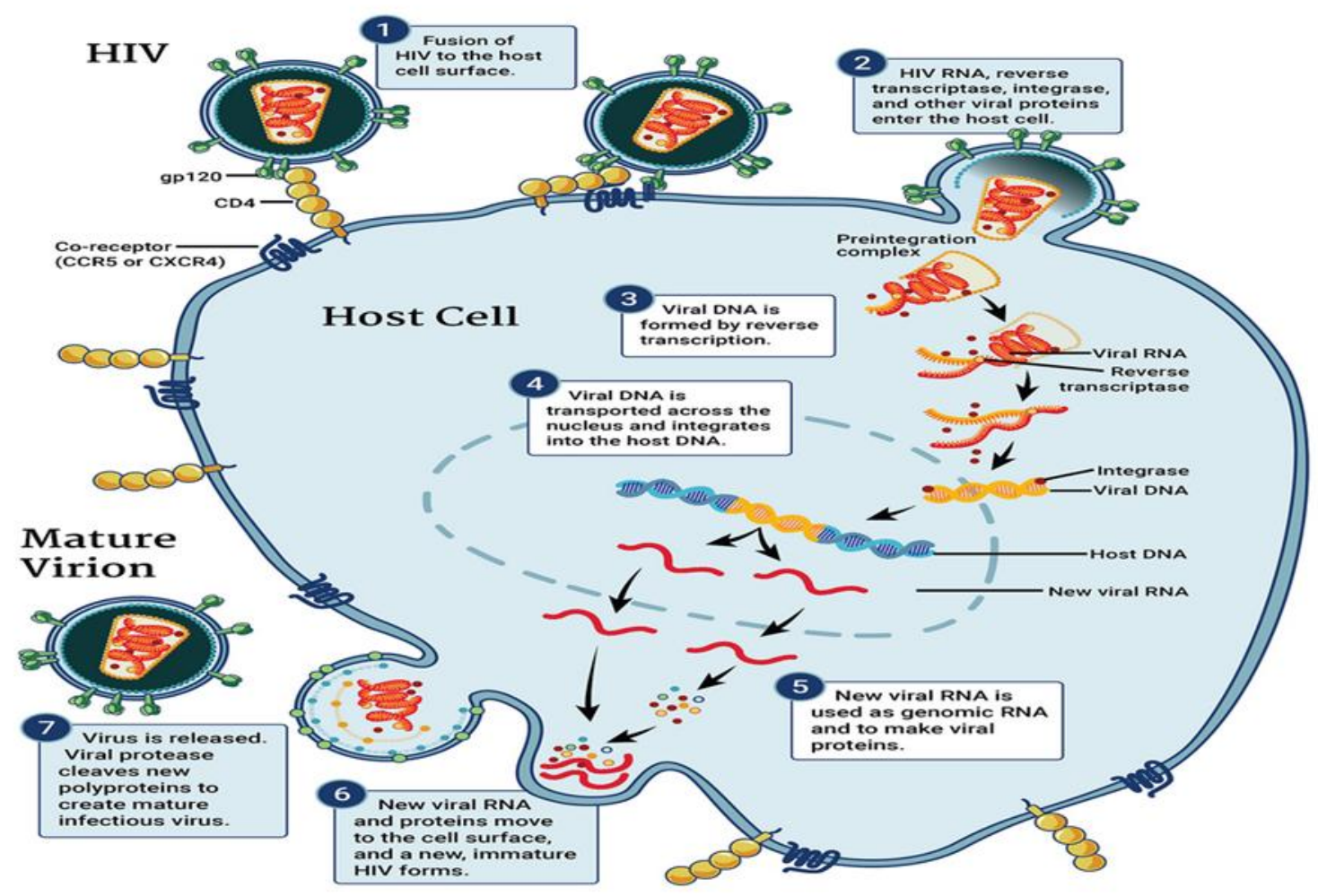

Fig. HIV Replication Cycle

Note: This illustration can be viewed on the NIAID website at www.niaid.nih.gov/diseases-conditions/hiv-replication-cycle

\section{UNITAID hosted and managed by the World Health Organization}

To increase the availability and accessibility of high-quality antiretrovirals, there is a growing demand for quality sheet diagnostics at affordable, reliable and guaranteed cost for use in resource-constrained settings [42]. Many argue that the POC diagnosis can be more scalable and can clarify the decentralized provision of antiretroviral treatment services at the community level. At the same time, simplify diagnosis - technologies can reduce the cost of diagnosis and reduce the follow-up of patients with HIV/AIDS, without compromising the quality of care, to understand the benefits of the diagnostic POC that can be made available; understand the overall vision of diagnostic technology. In order to maintain a high level of patient care, it is also important to examine the future perspectives of diagnosis and HIV. Increased efficiency can be achieved in terms of algorithm testing, cost of testing and decentralized service provision. application. Diagnoses are made during or near medical care. The initial assumption is that there is a need to significantly increase the level of access to high-quality, reliable diagnosis in resource-limited settings, as it is important to facilitate the early diagnosis and treatment of HIV/AIDS. AIDS, which will optimize prevention. antiretroviral therapy and help ensure an adequate and rapid response to drug resistance, a problem that is likely to worsen in the coming years. An optimal approach for better access to high-quality HIV/AIDS diagnostics is under development. Improved access is likely to be achieved through a combination of diagnostic services in most countries, which connect to the laboratory complex, high volume, low unit cost in high-density areas, and a platform for volume reduction. It is easier and much less populated settlement area. However, the best combination of technologies is unclear, and, in most countries, new distribution models may also emerge. At this stage, it is important that stakeholders, including the Ministry of Health, UNITAID, and other donors, seek to identify the most appropriate combination of investments to improve access to HIV diagnosis in settings limited resources for understanding diagnostic technologies. some products. This report analyzes current HIV diagnostic technologies, including: (i) the algorithms and tests needed to treat and treat HIV/AIDS before and after the start of treatment; (B) flat: the shapes used and the price of these tests; and (iii) test methods. Then, in the report, they are considered a modern diagnostic technique and a diagnostic platform for CD4 testing and viral load in adults and children and early infant diagnosis (EDI): three key areas of the test) (Including the viral load platform) is now available for all accessible laboratory-based test platforms, even under resource-constrained conditions. The report describes CD4 platforms, viral load, and EIS in markets and development, near and near POC, and analyzes the impact of landscape, including efficiency, can be achieved. Regarding test algorithms, there are costs of testing and decentralization. home delivery [42].

\section{Viral Load Technologies and Future Directions in Technology Technology Testing Unlike the CD4 test, in which even laboratory}

Systems have become the norm and have proven themselves with limited resources, this is not what you can see with the viral load test. Except for Brazil and South Africa, where viral load tests are generally conducted on a large scale, and in Brazil, which performs more than 1 million viral load tests each year and more than 2 million of viral load tests in South Africa in Botswana. and South Africa In Thailand, viral load tests have also been carried out on a relatively large scale. In addition, there is very little public health testing to determine the viral load under resource-constrained conditions. Some countries, including China, Kenya, and Lesotho, use all the viral load commonly used, but on a small scale. As mentioned above, this is partly done at the expense of infrastructure and the need for skilled technicians. However, since routine testing recommends systematic monitoring of viral load as the preferred method of the $2013 \mathrm{WHO}$ guidelines for diagnosing and confirming antiretroviral treatment failure, more and more countries are beginning to load reinforcement tests. viral. As for the CD4 test, you need to configure the transport networks so that the blood samples from the patients of a controlled laboratory are transferred to the analysis of the results and their transmission to patients in the suburbs and rural areas. For the patient Since viral load tests requiring plasma extraction, it is generally prescribed that whole blood samples have patients within six hours of their centrifugation. In addition, the plasma must be transported and stored in the refrigerator. These requirements put the sample transport system under pressure and contribute to the process. Introduction DBS on specific viral load laboratory platforms and its use as an EID test agent for better management of transfer process samples, whatever the time pressure [42].

Future Directions for the Study of the Influence of Viral Load and Viral Load of Increasing Consensus Technologies on the Importance of Viral Load Detection for Virologic Testing for Patients on Antiretroviral Therapy is likely to be a movement algorithm tests to routine viral stress tests, the frequency of testing is not yet determined, but if the simplicity of the tests and the cost allow, they could be as common in the future they are every few months or more often (similar to blood glucose in the diabetic test). Treatment with antiretrovirals, in general, should enable effective management and long-term chronic patients to ensure the successful treatment of as many people as possible. Early detection of viral resistance and reduction of treatment efficacy individually, resulting in a 
Citation: Mazen Eidah Alqurashi, et al, 2019. Characterization and molecular diagnosis of HIV/ADIS and its application. Journal of Applied Sciences Research., 15(5): 28-33. DOI: $10.22587 /$ jasr.2019.15.5.6

consequent improvement in compliance with the requirements of the existing treatment regimen or early detection of treatment failure to be protected to require modifications, are necessary to achieve this objective. Patient management algorithms need to be updated for the effective use of secure viral load information. As indicated in the context of the intensification of the CD4 test, the level of access required for the viral load test will probably be the establishment of a centralized test facility and a request prompting at the same time to use the DBS and POC tests. As mentioned above, the implementation of the technology is the SAMBA viral load limited in 2013, and the viral load and additional technologies VIZ POC are in the development phase, with the release of new products possible in 2014 and beyond. beyond its borders. It is too early to predict the exact price of equipment and POC tests. It is hoped, however, that the price per test will not exceed \$ 15 per test. Over time, competition between trading partners and third-party platforms may ultimately lead to prices such as the price level of CD4.

\section{What should the future diagnostic landscape of HIV look like?}

This report details the current situation of HIV diagnosis, from the detection of the virus to diagnosis and control of the disease in HIV patients. Given the importance attached to the effectiveness and simplification of antiretroviral therapy, consideration should be given to the adaptation and evolution of landscape diagnostics in the coming years in order to provide reliable, high-quality diagnostic services. effective, affordable and affordable for the necessary complementation of the tests necessary for the diagnosis, to organize and monitor effectively an HIV-positive patient. It can be argued that diagnostic services must be strategically provided, either centrally or under the control layer, using the most efficient, reliable and efficient technologies. The increase significantly improves the level of access to diagnosis, whether reliable or of high quality, plays a crucial role in (i) recognition and early treatment of HIV/AIDS, thus maximizing the preventive effect of HIV/ AIDS; treatment; (ii) early detection of drug resistance, thereby reducing the spread of drug-resistant virus strains; and (iii) maintain treatment plans, thereby extending the duration of successful treatment for each patient. While we have made substantial progress in expanding access to testing for the first HIV diagnosis, significant progress has been made in the diagnosis of the child, as well as in the planning and monitoring of antiretroviral therapy and new technologies in processing should lead to significant changes in the tests delivered. At the same time, they are also available, new and improved automated platforms for testing large volumes, offering cost-effective consolidation of testing in major centers. The speed with which the country will implement an optimized combination of centralized diagnostic services for the small amount of POC, designed to meet your individual needs, will determine the impact of these improved access, efficiency and quality technologies. home. in the next ten years. Further work to improve the diagnosis of HIV/AIDS should be addressed in several important areas. These include: improving quality at all levels of HIV/AIDS diagnostic tests; Analysis of the optimal combination of monitoring technologies taking into account country characteristics, identification of barriers to the introduction of new technologies and acceleration of their implementation, especially for POC technologies; Improvement of sample repositories and dissemination of results to central laboratories. Strategic funding from UNITAID and other donors could make a difference in many of these areas, including the introduction of new accelerated diagnostic technologies. UNITAID has committed to funding several projects to promote commercialization and support high-quality TOC diagnostics, including facilitating and accelerating their evaluation, approval, acceptance, and purchase to facilitate the widespread introduction of new technologies. Diagnostic [43].

\section{National Guidelines for HIV Testing, Overview of HIV Infection}

This chapter describes the history and current status of the HIV epidemic in India. It is defined as the structure, propagation, and classification of HIV. Immunopathogenesis and the factors that influence the progression of HIV infection are also discussed. On June 5, 1981, the US Centers for Disease Control and Prevention. UU. (CDC) [43] reported the identification of a new clinical entity called Acquired Immune Deficiency Syndrome in Canada. New York and California. Pneumocystis pneumonia (carinii), jiroveci and pneumonia (carinii) [44]. Rare skin cancer that usually occurs only in immunocompromised people. The agent that causes AIDS has been identified two years later. In 1986, the International Committee for the Taxonomy of Infected Aids for the Virus of Patients with AIDS isolated, the virus of the human immunodeficiency virus (HIV). The invasive, heterosexual and homosexual intercourse, through the transfusion of infected blood or through the sharing of needles and syringes and from mother to child. Because of this limited way, HIV is highly concentrated among high-risk groups. Those "high-risk groups" most likely to have sex workers (men and women), men who have sex with men, transgender people, and people who inject drugs [44].

\section{The current state of the epidemic: global and national.}

At the end of 2013, approximately 35 million (33.1 million - 37.2 million) people worldwide were infected with HIV. [45] The average prevalence among adults was $0.8 \%$. Of the total number of infections, 3.2 million occurred in children (under 15 years). Of these, 2.1 million new infections, reflecting a general decline in the number of new infections. Over a decade, 33 countries reported in decline in HIV incidence. Two-thirds of the country had a stable incidence rate. and the remaining third shows an increase in incidence. Over the last five years, the number of deaths from AIDS has decreased by 19\% [45]. The HIV/AIDS epidemic in India began in 1986-1987. Since the identification of the first HIV infection in Chennai and the first AIDS case in Mumbai. Since then, the HIV epidemic has spread to rural and urban areas, infecting high - risk populations and the general population. However, almost 25 years after the epidemic broke out in India, the disease did not reach levels predicted by experts from around the world. The Indian epidemic continues to be a concentrated epidemic with high HIV prevalence in the at-risk population. In 2012, the estimated prevalence among adults in total $0.27 \%$, and the total number of people living with HIV/AIDS was 20.89 lakhs [46-48]. Children under 15 years old accounted for 7\% (1.45 lakh). It is estimated that in 2011, 1,116,000 new HIV infections [4] were registered in India and approximately 14,500 new HIV infections per year in India.

\section{Virus: classification, structure, and genome}

HIV is a lentivirus that infects and destroys the cells of the immune system. Lentiviruses belong to a large group of viruses called retroviruses. The name "Lentivirus" means "slow virus". They are so named because they often last for many years until side effects occur on the body. There are two types of HIV: HIV1 and HIV-2. HIV-1 is the most common type in the world. HIV-2 has a limited geographical distribution. HIV-1 is closely related to the Monoimmuno-deficiency Virus (SIV) in distributed populations of wild chimpanzees in West Central Africa. HIV is an enveloped virus. The virus envelope consists of two layers of phospholipids, extracted from the membrane of the host cell. The envelope also contains trimers of the coated glycoprotein (GP) 160 protein. The Gp160 has two subunits, gp120 and gp41. While gp120 has an external protein and contains sites that bind to CD4 cells and central receptors on the surface of human CD4 T cells, gp41 is a membrane-bound protein. The viral envelope contains a layer called matrix, consisting of the p17 protein. The viral nucleus (or capsid) is generally in the form of a bullet and consists of a p24 protein. In the nucleus, there are three enzymes required for HIV replication: reverse transcriptase (RT), integrase and protease. The HIV genome also consists of two positive strands of single-stranded ribonucleic acid (RNA). The HIV genome is approximately $9.1 \mathrm{~kb}$ in size and contains nine genes and regions with terminal repeat length (LTR) at each end of the genome. Three structural genes code for virion structure proteins. These genes are called envelope (env), which encodes the HIV envelope. Antigen-specific groups (GAGs), which rapidly encode and die; and coding for DNA polymerase (Pol) incorporating the same protease and obvious enzymes RS and transcriptase. The regulatory genes required for viral replication are transcription activators (tat) as shown in Fig 1.1 and 1.2, [44,48]. 


\section{Figure 1.1. The structure of HIV}
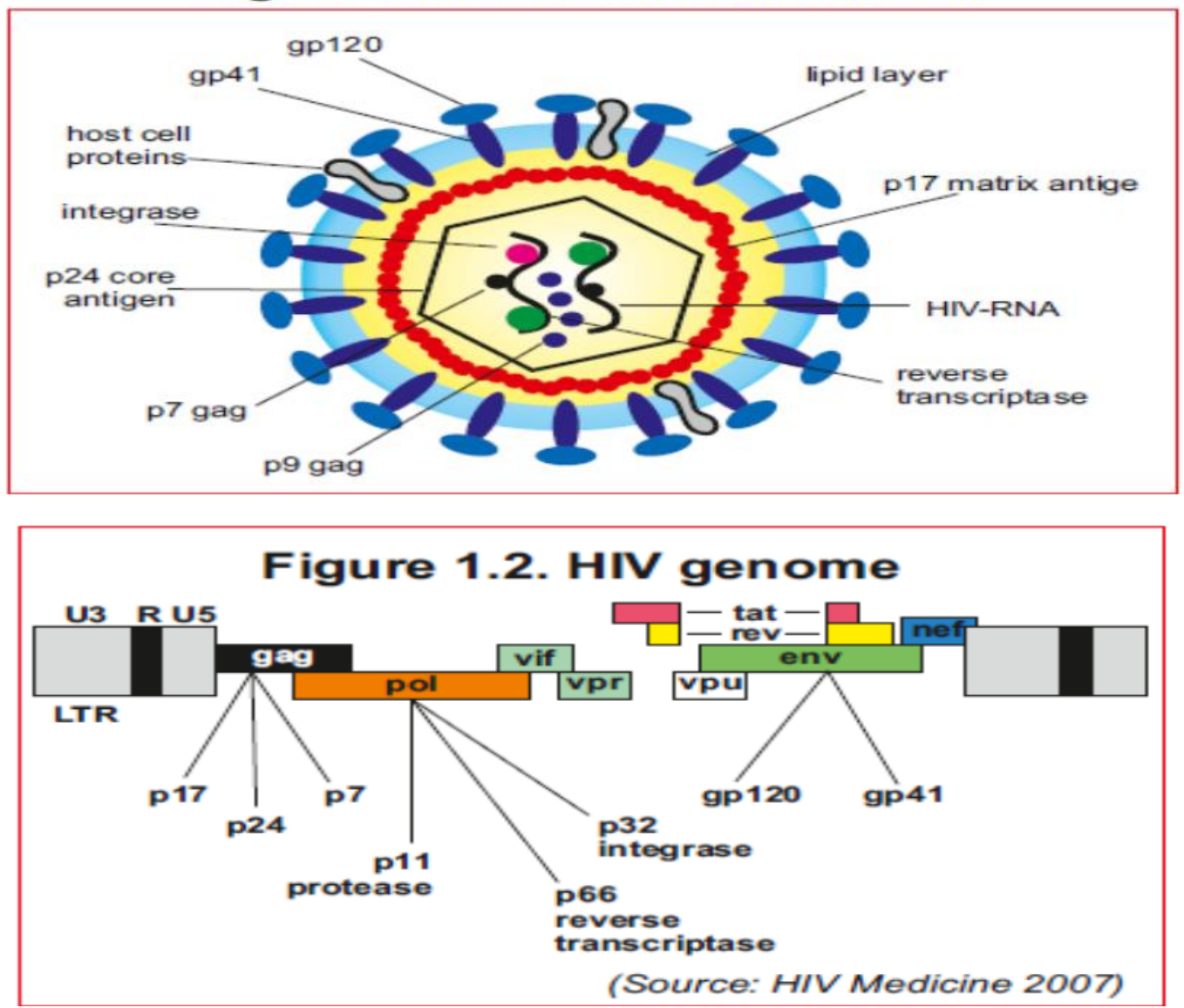

Ref. [44]

\section{CONFLICT OF INTEREST}

The authors declare no conflicts of interest.

\section{ACKNOWLEDGMENT}

The author support from molecular biology laboratory at King Fahd Center General Hospital, King Abdulaziz University, Jeddah City, Saudi Arabia

\section{REFERENCES}

[1] Centers for Disease Control and Prevention. HIV surveillance - the United States, 1981-2008. MMWR Morb Mortal Wkly Rep 2011; 60:689-93.

[2] US Preventive Services Task Force. Screening for HIV: recommendation statement. Issued July 2005, amended 2 April 2007. AHRQ Publication No. 070597-EF-2.

[3] Centers for Disease Control and Prevention. Revised recommendations for HIV testing of adults, adolescents, and pregnant women in healthcare settings. MMWR Recomm Rep 2006; 55(RR-14):1-17.

[4] World Health Organization. Towards universal access: scaling up priority HIV/AIDS interventions in the health sector: progress report. Geneva, Switzerland: WHO, 2010. Available at: Accessed 18 May 2012.

[5] Prejean J, Song R, Hernandez A, Ziebell R, et al. Estimated HIV incidence in the United States, 2006-2009. PLoS One 2011; 6:e17502.

[6] Clinical and Laboratory Standards Institute. Criteria for laboratory testing and diagnosis of human immunodeficiency virus infection: approved guideline. Document No. M53-A. Wayne, PA: CLSI, 2011.

[7] Brenner BG, Roger M, Routy JP, et al. High rates of forwarding transmission events after acute/early HIV-1 infection. J Infect Dis 2007; 195: 951-9.

[8] Wawer M J, Gray RH, Sewankambo NK, et al. Rates of HIV-1 transmission per coital act, by stage of HIV-1 infection, in Rakai, Uganda. J. Infect Dis 2005; 191:1403-9.

[9] Julia Kang Cornett and Thomas J. Kirn, Laboratory Diagnosis of HIV in Adults: A Review of Current Methods, Medical Microbiolog y, CID 2013:57.

[10] On the Fast-Track to an AIDS-Free Generation. The incredible journey of the global plan towards the elimination of new HIV infections among children by 2015 and keeping their mothers alive: Joint United Nations Programme on HIV/AIDS; 2016.

[11] Violari A, Cotton MF, Gibb DM, Babiker AG, Steyn J, Madhi SA, et al. Early antiretroviral therapy and mortality among HIV-infected infants. N Engl J Med. 2008;359(21):2233-44.

[12] Global plan Kenya Country fact sheet: UNAIDS; 2016

[13] United Nations Children's Fund. UNICEF Data: Paediatric Care and Treatment, Early Infant HIV Diagnosis downloadable spreadsheet. 2018.Accessed 17 Jan 2019.

[14] Ciaranello AL, Park JE, Ramirez-Avila L, Freedberg KA, Walensky RP, Leroy V. Early infant HIV-1 diagnosis programs in resource-limited settings: opportunities for improved outcomes and more cost-effective interventions. BMC Med. 2011; 9:59.

[15] Team A, Gill MM, Hoffman HJ, Isavwa A, Mokone M, Foso M, et al. Conventional early infant diagnosis in Lesotho from specimen collection to results usage to manage patients: where are the bottlenecks? PLoS One. 2017;12(10): e0184769.

[16] Sutcliffe CG, van Dijk JH, Hamangaba F, Mayani F, Moss WJ. Turnaround time for early infant HIV diagnosis in rural Zambia: a chart review. PLoS One. 2014;9(1): e87028.

[17] Lankowski AJ, Siedner MJ, Bangsberg DR, Tsai AC. Impact of geographic and transportation-related barriers on HIV outcomes in sub-Saharan Africa: a systematic review. AIDS Behav. 2014;18(7):1199-223. 
Citation: Mazen Eidah Alqurashi, et al, 2019. Characterization and molecular diagnosis of HIV/ADIS and its application. Journal of Applied Sciences Research., 15(5): 28-33. DOI: $10.22587 /$ jasr.2019.15.5.6

[18] Essajee S, Vojnov L, Penazzato M, Jani I, Siberry GK, Fiscus SA, et al. Reducing mortality in HIV-infected infants and achieving the 90-90-90 target through innovative diagnosis approaches. J Int AIDS Soc. 2015; 18(Suppl 6):20299.

[19] Gill MM, Mofenson LM, Phalatse M, Tukei V, Guay L, Nchephe M. Piloting very early infant diagnosis of HIV in Lesotho: acceptability and feasibility among mothers, health workers, and laboratory personnel. PLoS One. 2018; 13(2): e0190874.

[20] Lilian RR, Kalk E, Bhowan K, Berrie L, Carmona S, Technau K, et al. Early diagnosis of in utero and intrapartum HIV infection in infants prior to 6 weeks of age. J Clin Microbiol. 2012;50(7):2373-7.

[21] Lilian RR, Kalk E, Technau KG, Sherman GG. Birth diagnosis of HIV infection in infants to reduce infant mortality and monitor for the elimination of mother-to-child transmission. Pediatr Infect Dis J. 2013;32(10):1080-5.

[22] Finocchario-Kessler S, Gautney BJ, Khamadi S, Okoth V, Goggin K, Spinler JK, et al. If you text them, they will come using the HIV infant tracking system to improve early infant diagnosis quality and retention in Kenya. AIDS. 2014; 28(Suppl 3): S313-21.

[23] Kayumba K, Nsanzimana S, Binagwaho A, Mugwaneza P, Rusine J, Remera E, et al. TRACnet internet and short message service technology improves time to antiretroviral therapy initiation among HIV-infected infants in Rwanda. Pediatr Infect Dis J. 2016;35(7):767-71.

[24] Phiri NA, Lee HY, Chilenga L, Mtika C, Sinyiza F, Musopole O, et al. Early infant diagnosis and outcomes in HIV-exposed infants at a central and a district hospital, northern Malawi. Public Health Action. 2017;7(2):83-9.

[25] Bourne DE, Thompson M, Brody LL, Cotton M, Draper B, Laubscher R, et al. Emergence of a peak in early infant mortality due to HIV/AIDS in South Africa. AIDS. 2009;23(1):101-6.

[26] International NBoS-KaI. 2014 Kenya demographic and health survey key findings. Rockville, Maryland: KNBS and ICF International. p. 2015.

[27] Consolidated guidelines on the use of antiretroviral drugs for treating and preventing HIV infection: recommendations for a public health approach - 2nd ed: World Health Organization; 2016.

[28] Ministry of Health NASCP. Guidelines on the use of antiretroviral drugs for treating and preventing HIV infection in Kenya 2016. Nairobi: NASCOP; 2016.

[29] Chiu A, Modi S, Rivadeneira ED, Koumans EH. Optimizing infant HIV diagnosis in resource-limited settings: modeling the impact of HIV DNA PCR testing at birth. J Acquir Immune Defic Syndr. 2016;73(4):454-62.

[30] Celletti F, Sherman G, Mazanderani AH. Early infant diagnosis of HIV: a review of current and innovative practices. Curr Opin HIV AIDS 2017;12(2):112-6.

[31] WHO Prequalification of In Vitro Diagnostics Public Report. Product: Xpert® HIV-1 Qual Assay WHO reference number: PQDx 0259-070-00: World Health Organization; 2016.

[32] WHO Prequalification of In Vitro Diagnostics Public Report. Product: Alere ${ }^{\mathrm{TM}}$ q HIV-1/2 Detect WHO reference number: PQDx 0226-032-00: World Health Organization; 2016.

[33] Hsiao NY, Dunning L, Kroon M, Myer L. Laboratory evaluation of the Alere q point-of-care system for early infant HIV diagnosis. PLoS One. 2016;11(3): e0152672.

[34] Ibrahim M, Moyo S, Mohammed T, Mupfumi L, Gaseitsiwe S, Maswabi K, et al. Brief report: high sensitivity and specificity of the Cepheid Xpert HIV-1 qualitative point-of-care test among newborns in Botswana. J Acquir Immune Defic Syndr. 2017;75(5): e128-e31.

[35] Technau KG, Kuhn L, Coovadia A, Murnane PM, Sherman G. Xpert HIV-1 point-of-care test for neonatal diagnosis of HIV in the birth testing program of a maternity hospital: a field evaluation study. Lancet HIV. 2017;4(10): e442-e8.

[36] Murray TY, Sherman GG, Nakwa F, MacLeod WB, Sipambo N, Velaphi S, et al. Field evaluation of the performance of Alere and Cepheid qualitative HIV assays for pediatric point-of-care testing in an Academic Hospital in Soweto, South Africa. J Clin Microbiol. 2017;55(11):3227-35.

[37] Jani IV, Meggi B, Mabunda N, Vubil A, Sitoe NE, Tobaiwa O, et al. Accurate early infant HIV diagnosis in primary health clinics using a point-of-care nucleic acid test. J Acquir Immune Defic Syndr. 2014;67(1): e1-4.

[38] Dunning L, Kroon M, Hsiao NY, Myer L. Field evaluation of HIV point-of-care testing for early infant diagnosis in Cape Town, South Africa. PLoS One. 2017;12(12): e0189226.

[39] Finocchario-Kessler S, Goggin K, Khamadi S, Gautney B, Dariotis JK, Bawcom C, et al. Improving early infant HIV diagnosis in Kenya: study protocol of a cluster-randomized efficacy trial of the HITSystem. Implement Sci. 2015; 10:96.

[40] National AIDS Control Council. Kenya AIDS Response Progress Report 2016. 2016.

[41] Matthew R. Sandbulte, Brad J. Gautney, May Maloba, Catherine Wexler, Melinda Brown, Natabhona Mabachi, Kathy Goggin, Raphael Lwembe6, Niaman Nazir, Thomas A. Odeny and Sarah Finocchario-Kessler Ab Infant HIV testing at birth using point-of-care and conventional HIV DNA PCR: an implementation feasibility pilot study in Kenya, BMC(2019) 5:18.

[42] WHO, UNITAID is hosted and administered by the World Health Organization, HIV/AIDS Diagnostics Technology Landscape $4^{\mathrm{TH}}$ EDITION, June 2014,1147.

[43] CDC, 1993 revised classification system for HIV infection and expanded surveillance case definition for AIDS among adolescents and adults. MMWR Recomm Rep. 1992. 41: p. 1-19.

[44] NACO MH, National AIDS Control Organization Ministry of Health \& Family Welfare, Government of India, 2015. National Guidelines for HIV Testing, Chapter 10 1-120.

[45] Gottlieb, M.S., et al., Pneumocystis carinii pneumonia and mucosal candidiasis in previously healthy homosexual men: evidence of a new acquired cellular immunodeficiency. N Engl J Med, 1981. 305(24): p. 1425-31.

[46] UNAIDS, UNAIDS Report on the Global AIDS epidemic. 2010.

[47] NACO, NACO Annual Report 2012-13. 2012-13.

[48] Requejo, H.I., Worldwide molecular epidemiology of HIV. Rev Saude Publica, 2006. 40(2): p. 331-45 